\title{
Fuzzy logic: adding natural uncertainties into environmental assessment
}

\author{
Amorim, L. G. B.*; Massone, C. G.; WAgener, A. R. L.
}

Chemistry Department, Pontifícia Universidade Católica do Rio

de Janeiro, 22453-900 Rio de Janeiro, Brazil

(*correspondence: leogrippbom@gmail.com)

This research aimed at testfying the possible advantages of using of Fuzzy logic as opposed to boolean logic to assess environmental contamination. Results from traditional assessment tools obtained for a tropical area [1] were compared with those derived from the Fuzzy Logic, by Fuzzy C-Means algorithm (FCM). This approach proved to be efficient as for the qualitative aspect of the environmental contamination analysis since it is unlikely that a sample will be composed exclusively of a single contaminant source. The gradients created by the FCM method show the classes values overlapped in the space. The greater complexity aggregates natural uncertainty and has higher reliability compared to traditional methods that interpolate as singles sources (Figure 1). From this approach it was possible to realize new data interpretations, more appropriate for environmental management focus.

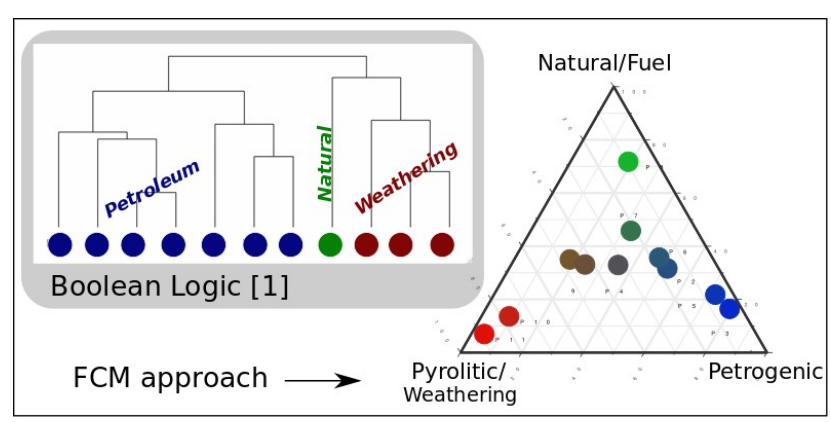

Figure 1 - Representation of the degrees of pertinence of the samples in relation to Natural/Fuel, Petrogenic and Pyrolitic groups.

[1] Massone et al. (2013) Marine Pollution Bulletin, 73(1), 345354 